\title{
Efficacy of cryoablation in idiopathic and non-idiopathic atrial fibrillation patients
}

\author{
Boho $\mathrm{A}^{1,2}$, Stancak $\mathrm{B}^{1,2}$, Misikova $\mathrm{S}^{1,2}$ \\ East Slovakia Institute for Cardiac and Vascular Diseases, Kosice, Slovakia. smisikova@vusch.sk
}

\section{ABSTRACT}

OBJECTIVES AND BACKGROUND: Studies assessing the unicentric and prospective comparison of efficacy of cryoballoon pulmonary vein isolation in idiopathic and non-idiopathic atrial fibrillation (AF) patients are missing. The aim of this study was to compare a single procedural outcome in these subgroups of AF patients.

METHODS: A total of 208 patients with drug resistant AF were included in this study. Among them, 36 patients had idiopathic AF and 172 patients had non-idiopathic AF. The efficacy endpoint was freedom from any atrial arrhythmia lasting $>30 \mathrm{~s}$ after a 3-months blanking period.

RESULTS: The freedom from atrial arrhythmias without antiarrhythmic drugs after 1 and 3 years of follow-up, respectively, was $85.2 \%$ and $70.4 \%$ in patients with idiopathic AF; and $64.6 \%$ and $39.9 \%$ in patients with non-idiopathic AF $(p=0.021)$. Moreover, the success rate when analysing only paroxysmal AF patients after 1 and 3 years of follow-up, respectively, was $95.7 \%$ and $82 \%$ in patients with idiopathic AF; and $72.6 \%$ and $47 \%$ in patients with non-idiopathic AF $(p=0.022)$.

CONCLUSIONS: A single cryoballoon pulmonary vein isolation was more effective in preventing atrial arrhythmias relapses in idiopathic AF patients compared to non-idiopathic AF patients. The best efficacy outcomes were observed in patients with paroxysmal idiopathic AF (Tab. 5, Fig. 2, Ref. 25). Text in PDF www.elis.sk

KEYWORDS: catheter ablation, cryoballoon, lone atrial fibrillation, idiopathic atrial fibrillation.

\section{Introduction}

Atrial fibrillation (AF) is the most common sustained cardiac arrhythmia and is independently associated with a two-fold increased risk of all-cause mortality in women and a 1.5-fold increase in men (1). Over the years, pulmonary vein isolation (PVI) has become the cornerstone in the therapy for drug-refractory paroxysmal and persistent AF $(1,2)$. Nowadays, the improved second-generation cryoballoon (CB) catheter is increasingly being used for the treatment of AF because of its proven efficacy that is non-inferior to conventional point-by-point ablation techniques $(3,4)$. Moreover, less serious complications and ideally achieved PVI with a single application are other undeniable advantages of CB PVI $(5,6)$.

Most AF patients have one or more cardiovascular comorbidities, while there is only a minority of patients, who develop AF without any obvious structural heart disease. To refer to this

\footnotetext{
${ }^{1}$ Cardiology Clinic, Arrhythmology Section, East Slovakia Institute for Cardiac and Vascular Diseases, Kosice, Slovakia, and ${ }^{2}$ Pavol Jozef Safarik University, Faculty of Medicine, Kosice, Slovakia
}

Address for correspondence: S. Misikova, Institutional address: East Slovakia Institute for Cardiac and Vascular Diseases, Ondavska 8, SK- 04011 Kosice, Slovakia.

Phone: +421557891310 , Fax: +421557891313 group of patients, the term "lone" AF was firstly introduced in 1953 (7). Current views on lone AF already differ in many ways. Although the term "lone AF" is considered obsolete nowadays, in routine clinical practice it is still used to address younger AF patients without the echocardiographic and clinical evidence of cardiovascular, pulmonary or endocrine diseases.

There are only limited data on mid- to long-term efficacy of CB PVI in patients with lone AF. Moreover, to the best of our knowledge, data assessing the direct and unicentric comparison of efficacy of CB PVI in ,idiopathic” and „,non-idiopathic“ AF patients are missing. Therefore, we hereby present our findings of a single procedural outcome of CB PVI in these subgroups of AF patients.

\section{Materials and methods}

\section{Study population}

This unicentric study prospectively followed the patients scheduled for PVI using second-generation CB catheter in our institute during 2014-2017. All the patients provided an informed consent prior to the procedure. Patients involved had a highly symptomatic and drug-resistant paroxysmal or persistent AF, defined according to the current guidelines. Only patients without prior ablations for AF were included in this study. General inclusion and exclusion criteria were reported previously (5). 
Tab. 1. Factors and diseases excluding the diagnosis of idiopathic atrial fibrillation.

Age $>60$ years

Arterial hypertension

Coronary heart disease

Chronic heart failure

Valvular heart disease

Structural heart disease

Chronic obstructive pulmonary disease

Bronchial asthma

Obstructive sleep apnea

Diabetes mellitus

Thyroid disease

Alcohol abuse

For the purpose of this study, as the idiopathic atrial fibrillation (IAF) patients were considered individuals not older than 60 years, without any (echocardiographic or clinical) evidence of cardiovascular, cardiopulmonary or structural heart disease and also diabetes mellitus. The presence of isolated left atrial (LA) enlargement on echocardiography was not considered as structural heart disease and thus not excluded in the diagnosis of IAF. LA enlargement may be either a cause or a consequence of AF (8). The monitored diseases or factors in relation to the patients with IAF are listed in the Table 1.

\section{Ablation procedure}

Prior to the procedure, a computed tomography was used to obtain the information on the anatomy of LA in all the patients. Cryoballoon ablation procedures were performed as was previously described in detail $(3,9)$. Briefly, procedures were performed under a conscious sedation, with a routine use of intracardiac echocardiography to exclude thrombi in LA and to guide the transseptal puncture. Heparin was administered after the transseptal puncture and activated clotting time was maintained $>300 \mathrm{~s}$ during the whole procedure. After gaining LA Access, through a steerable 15Fr sheath (FlexCath Advance, Medtronic), a $28 \mathrm{~mm} \mathrm{CB}$ (Arctic Front Advance Cryoballoon Catheter, Medtronic) was advanced, and using an inner lumen-mapping catheter (Achieve, Medtronic), $\mathrm{CB}$ was positioned and inflated sequentially at the ostium of each pulmonary vein (PV). Applications of cryothermal energy lasted 240 s. PVI was confirmed using the Achieve mapping catheter if PV potentials completely disappeared or were dissociated from LA activity after the applications. A,,bonus freezes” ( 1 or 2 depending on temperature reached) were delivered after PVI confirmation. In the case when a successful isolation of PV could not be achieved a ,pull down" technique was utilized (9). To monitor phrenic nerve function and to prevent phrenic nerve palsy (PNP) during the isolation of right-sided PVs, pacing the ipsilateral phrenic nerve was achieved by a quadripolar catheter positioned in the superior vena cava. Capture was confirmed by palpation of the right hemidiaphragmatic contractions along with CMAP (compound motor action potential) monitoring (10).

\section{Follow up and data collection}

The hospitalization typically lasted 48 hours following the cryoablation. All the patients were scheduled for ambulatory fol- low-up visits in our institute 3 times a year. Late-term follow-up ( $>1$ year after PVI) was performed twice a year. The assessment of AF recurrence was executed via analysis of patient's clinical history, serial ECG recordings and Holter ECG recordings. Holter follow-up comprised 24-hour Holter monitoring at least twice a year, and 10 or 14-days Holter monitoring once a year. Moreover, all the patients were encouraged to have a twelve lead ECG performed in any case of cardiac palpitations and to contact our institute. Any not clear, but potentially suspicious symptoms of AF that were not captured on the ECG, were deemed as deserving an additional Holter monitoring. An arrhythmia recurrence was defined as a symptomatic or documented (both symptomatic and asymptomatic) episode of AF, atrial flutter or atrial tachycardia lasting $>30 \mathrm{~s}$. A blanking period of 3 months after the procedure

Tab. 2. Baseline characteristics of study population.

\begin{tabular}{|c|c|c|}
\hline & Mean \pm SD/Proportion & Median (IQR) \\
\hline Age (years) & $57 \pm 9.4$ & $58(29-74)$ \\
\hline Males ( \%) & 70.2 & - \\
\hline Body height $(\mathrm{cm})$ & $176 \pm 10.1$ & $176(153-202)$ \\
\hline Body weight $(\mathrm{kg})$ & $91 \pm 13.2$ & $91(52-128)$ \\
\hline $\operatorname{BSA}\left(m^{2}\right)$ & $2.1 \pm 0.2$ & $2.1(1.5-2.6)$ \\
\hline $\mathrm{BMI}\left(\mathrm{kg} / \mathrm{m}^{2}\right)$ & $29.4 \pm 3.7$ & $29.3(20.8-42.9)$ \\
\hline LA dilated $(\%)$ & 57.2 & - \\
\hline LV diameter (mm) & $49 \pm 4.9$ & $49(36-63)$ \\
\hline LV ejection fraction $(\%)$ & $57.5 \pm 6.3$ & $60(35-70)$ \\
\hline Mitral regurgitation $\geq 2$ nd gr. $(\%)$ & 4.8 & - \\
\hline Paroxysmal AF (\%) & 61.5 & - \\
\hline Idiopathic AF (\%) & 17.3 & - \\
\hline Hypertension (\%) & 70.2 & - \\
\hline Diabetes mellitus (\%) & 8.7 & - \\
\hline CAD/PAD (\%) & $7.7 / 3.4$ & - \\
\hline Previous stroke or TIA (\%) & 9.1 & - \\
\hline $\mathrm{CHA}_{2} \mathrm{DS}_{2}$-VASc score & $2 \pm 1.2$ & $1(0-6)$ \\
\hline
\end{tabular}

$\mathrm{AF}$ - atrial fibrillation, $\mathrm{BMI}$ - body mass index, BSA - body surface area, $\mathrm{CAD}-$ coronary artery disease, IQR - interquartile range, LA - left atrium, LV - left ventricle, PAD - peripheral artery disease, TIA - transitory ischemic attack

Tab. 3. Procedural characteristics.

\begin{tabular}{ll}
\hline & Mean \pm SD/Proportion \\
\hline Total laboratory occupancy time $(\mathrm{min})$ & $163 \pm 39$ \\
\hline Total fluoroscopy time $(\mathrm{min})$ & $17.8 \pm 6.2$ \\
\hline Normal PV anatomy, $\mathrm{n}$ of patients $(\%)$ & $194(93.3)$ \\
\hline Left common ostium, $\mathrm{n}$ of patients $(\%)$ & $12(5.8)$ \\
\hline Accessory PV, $\mathrm{n}$ of patients (\%) & $2(1)$ \\
\hline Acute isolation of PV, $\mathrm{n}(\%)$ & $796(96.8)$ \\
\hline Number of cryoballoon applications & \\
LSPV & $3.3 \pm 1.6$ \\
LIPV & $3.2 \pm 1.5$ \\
RSPV & $2.6 \pm 1.1$ \\
RIPV & $2.6 \pm 1.4$ \\
\hline Temperature reached during cryoablation $\left({ }^{\circ} \mathrm{C}\right)$ & \\
LSPV & $-50 \pm 8$ \\
LIPV & $-44 \pm 12$ \\
RSPV & $-52 \pm 9$ \\
RIPV & $-46 \pm 11$ \\
\hline
\end{tabular}

LIPV - left inferior pulmonary vein, LSPV - left superior pulmonary vein, PV - pulmonary vein, RIPV - right inferior pulmonary vein, RSPV - right superior pulmonary vein 
was applied in this study. After the blanking period, class I or III antiarrhythmic drugs (AADs) were discontinued.

All procedure-related adverse events were systematically recorded and divided into major and minor categories. Major complications were considered those requiring an intervention or leading to a permanent health injury, or which necessitated a prolonged hospitalization exceeding 48 hours after the procedure.

\section{Statistical analysis}

Statistical analyses were performed using the SPSS Statistics, Version 20.0 (IBM). Categorical variables were presented as the percentages and analysed by chi-square test with Yates's correction. Continuous variables were expressed as the mean \pm standard deviation or median and interquartile range as appropriate and analysed by the Student's $t$-test. Kaplan-Meier analysis was used to estimate arrhythmia free survival after a 90-day blanking period and log rank test was applied to determine any significant difference. Univariate and multivariate analyses using the Cox regression model were applied to identify factors predictive of arrhythmia recurrence. A two-tailed $p$ value $<0.05$ was considered statistically significant.

\section{Results}

\section{Patients and procedural characteristics}

A total of 208 patients ( $70 \%$ men, mean age $57 \pm 9.4$ years) were enrolled in this study. All the patients had failed at least 1 AAD. Thirty-six of them met the criteria of IAF. Baseline patient's characteristics are summarized in the Table 2. All the patients underwent the PVI using the large $28 \mathrm{~mm} \mathrm{CB}$ catheter. The mean total laboratory occupancy time was $163 \mathrm{~min}$. The mean total fluoroscopy time was $17.8 \mathrm{~min}$. Normal PV anatomy was found in 194 (93.3\%) of the patients. Electrical isolation could be achieved in $96.8 \%$ of PVs (796 of 822). Procedural details are presented in the Table 3. Characteristics of the study subgroups are shown in

Tab. 4. Characteristics of study subgroups.

\begin{tabular}{|c|c|c|c|}
\hline & $\begin{array}{l}\text { Idiopathic AF } \\
(\mathrm{n}=36)\end{array}$ & $\begin{array}{l}\text { Non-idiopathic AF } \\
(\mathrm{n}=172)\end{array}$ & $\mathrm{p}$ \\
\hline Age (years) & $50.2 \pm 10$ & $57.9 \pm 8.7$ & $<0.001$ \\
\hline Males (\%) & 69.5 & 70.3 & 0.93 \\
\hline Body height (cm) & $178.8 \pm 11,7$ & $175.3 \pm 9.7$ & 0.06 \\
\hline Body weight (kg) & $90.6 \pm 14.6$ & $91.1 \pm 13$ & 0.84 \\
\hline $\operatorname{BSA}\left(\mathrm{m}^{2}\right)$ & $2.08 \pm 0.2$ & $2.07 \pm 0.2$ & 0.49 \\
\hline BMI $\left(\mathrm{kg} / \mathrm{m}^{2}\right)$ & $28.4 \pm 3.7$ & $29.6 \pm 3.6$ & 0.06 \\
\hline LA dilated (\%) & 44.4 & 59.3 & 0.15 \\
\hline LV diameter (mm) & $50.6 \pm 5.4$ & $48.9 \pm 4.8$ & 0.34 \\
\hline Paroxysmal AF (\%) & 75 & 58.7 & 0.1 \\
\hline LV ejection fraction (\%) & $59 \pm 4$ & $57.6 \pm 7.9$ & 0.38 \\
\hline Hypertension (\%) & 0 & 84.3 & $<0.001$ \\
\hline Diabetes mellitus (\%) & 0 & 10.5 & 0.09 \\
\hline CAD or PAD $(\%)$ & 0 & 13.4 & 0.04 \\
\hline Previous stroke or TIA (\%) & 5.6 & 9.9 & 0.6 \\
\hline $\mathrm{CHA}_{2} \mathrm{DS}_{2}$-VASc score & $0.6 \pm 0.9$ & $1.8 \pm 1.2$ & $<0.001$ \\
\hline Normal PV anatomy, $\mathrm{n}$ of patients $(\%)$ & $34(94.4)$ & $160(93)$ & 0.96 \\
\hline Acute isolation of $\mathrm{PV}, \mathrm{n}(\%)$ & $139(97.9)$ & $657(96.6)$ & 0.6 \\
\hline
\end{tabular}

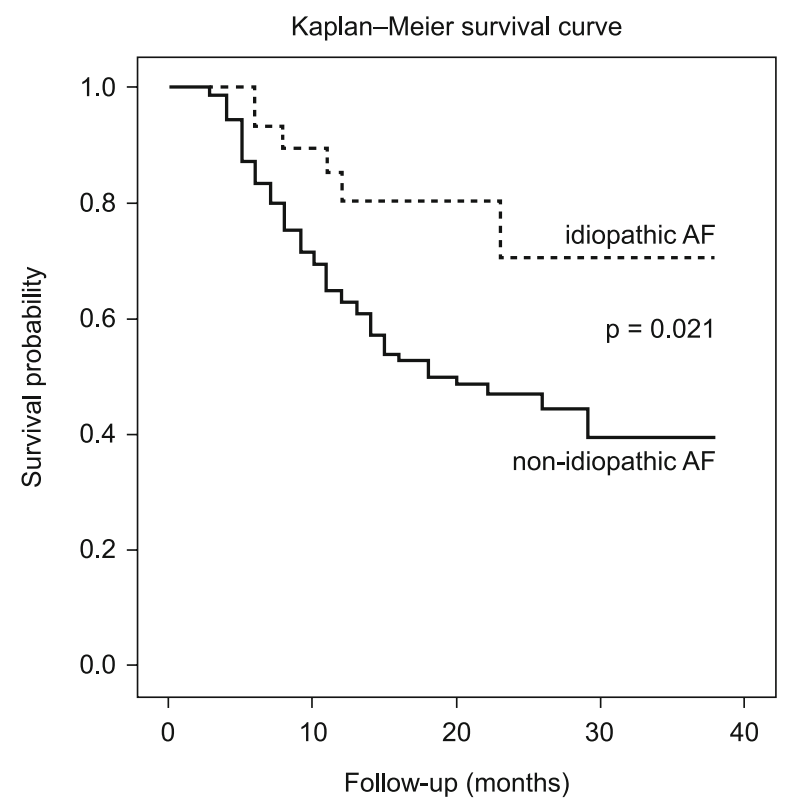

Fig. 1. Comparison of efficacy outcomes in patients with idiopathic and non-idiopathic atrial fibrillation (AF).

the Table 4. Of note, patients with IAF were significantly younger ( 50.2 vs 57.9 years, $\mathrm{p}<0.001)$. Other statistically significant differences between the two subgroups were related to associated diseases, but this resulted from the study design.

\section{Efficacy outcomes}

During the 36-months follow-up, 11 patients (5.3\%) were lost, most of them within the first year after the procedure. Following a single CB PVI, the incidence rates of atrial arrhythmias recurrence at 12 and 36 months of follow-up, respectively, was $14.8 \%$ and $29.6 \%$ in IAF group and $35.4 \%$ and $60.1 \%$ in non-IAF group, respectively (log rank, $\mathrm{p}=0.021$ ) (Fig. 1$)$. The median AF-free survival times in IAF and non-IAF groups were $>36$ months and 18 months, respectively.

There were 128 (61.5\%) patients with paroxysmal AF in this study. Twenty-seven had IAF, while 101 had non-IAF. When comparing the efficacy outcomes only in paroxysmal AF patients at 12 and 36 months of follow-up, respectively, $95.7 \%$ and $82 \%$ of IAF patients and $72.6 \%$ and $47 \%$ of nonIAF patients, respectively, remained free of documented atrial arrhythmias (log rank, $\mathrm{p}=0.022$ ) (Fig. 2).

Multivariate Cox regression model revealed that persistent AF and dilated LA were statistical significant predictors predisposing to arrhythmia recurrence in nonIAF patients with $\sim 2$-fold risk for persistent AF (HR 1.74; $95 \%$ CI: 1.03-2.94, p= 


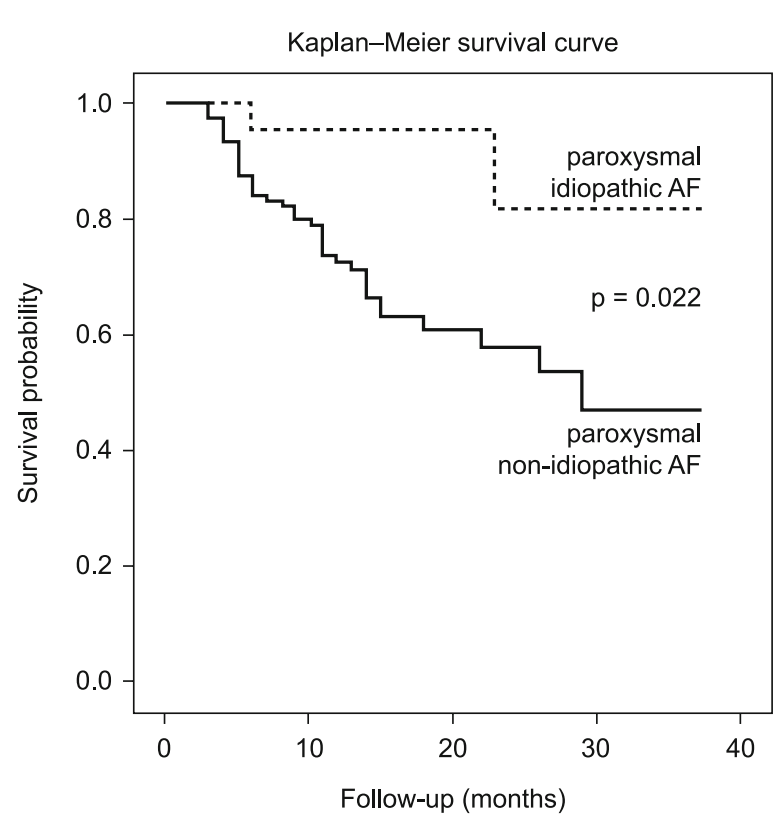

Fig. 2. Comparison of efficacy outcomes in patients with paroxysmal idiopathic and paroxysmal non-idiopathic atrial fibrillation (AF).

0.037 ) as well as for dilated LA (HR 2.25; $95 \%$ CI: 1.26- .02, $\mathrm{p}=0.006$ ). Although in the univariate analysis the persistent AF correlated with arrhythmia recurrences in IAF study subgroup, it did not meet the criteria for statistical significance in the multivariate analysis.

\section{Complications}

Procedure-related complications were recorded in 15 (7.2\%) patients, however as the major complications were considered 4 $(1.9 \%)$ of them (Table 5$)$. The most common adverse event was PNP, which occurred in $8(\sim 3.9 \%)$ patients during the right superior ( 7 cases) and right inferior ( 1 case) PV ablation. Most of the PNPs (6 of 8$)$ were transient and recovered within the procedure. Of the remaining PNPs, one resolved prior to discharge from the hospital and the last one within 12 months following the procedure. Two patients $(\sim 1 \%)$ had a pericardial effusion, one of them required a pericardiocentesis. In 5 patients $(2.4 \%)$, groin complications were observed, including 2 arteriovenous fistulas and 3 cases of pseudoaneurysm. Two of the groin complications were treated surgically. The most important severe complications like death, stroke/TIA or atrioesophageal fistula were not clinically recorded.

Tab. 5. Procedure-related adverse events.

\begin{tabular}{lccc}
\hline & Minor n (\%) & Major $\mathrm{n}(\%)$ & Overall $\mathrm{n}(\%)$ \\
\hline Phrenic nerve palsy & $7(3.37)$ & $1(0.48)$ & $8(3.85)$ \\
Pericardial effusion & $1(0.48)$ & $1(0.48)$ & $2(0.96)$ \\
Arteriovenous fistula & $1(0.48)$ & $1(0.48)$ & $2(0.96)$ \\
Pseudoaneurysm & $2(0.96)$ & $1(0.48)$ & $3(1.44)$ \\
Overall & $11(5.29)$ & $4(1.92)$ & $15(7.21)$ \\
\hline
\end{tabular}

\section{Discussion}

To the best of our knowledge, this is the first unicentric study comparing directly the long-term follow-up efficacy of CB PVI in patients with IAF and non-IAF. In general, we observed lower success rates of $\mathrm{CB}$ procedures for non-IAF patients than for IAF patients, as well as for persistent $\mathrm{AF}$ than for paroxysmal AF. The main finding is that PVI using CB catheter in younger IAF patients is highly effective and safe.

Efficacy and safety outcomes

In our study, the freedom from atrial arrhythmias without AADs in IAF patients after 1 and 3 years of follow-up, respectively, was $85.2 \%$ and $70.4 \%$; and $95.7 \%$ and $82 \%$ when analysed only paroxysmal IAF patients. There were only few studies that evaluated the outcome after CB PVI in specific group of IAF patients. The largest was the study of Wójcik et al (11), reporting 103 patients with a paroxysmal lone $\mathrm{AF}$ treated by $\mathrm{CB}$ technique. There was $\sim 90 \%$ and $77 \%$ success rate after a single CB PVI without AADs during 1 and 5 years of follow-up, respectively. It is worth mentioning, that in up to $18 \%$ of patients, the use of either 2 balloon sizes or focal tip catheter was necessary to achieve a complete PVI. In another study of Buiatti et al (12), of 76 patients with lone AF (both paroxysmal and persistent) 56 (78\%) were free from arrhythmia relapses without AADs after the mean follow-up of $458 \pm 344$ days. Of note, in patients with persistent lone AF either CFAE ablation or linear lesions were performed additionally to CB PVI. The report of De Regibus et al (13) outlined 75 patients with paroxysmal lone AF, who underwent a single $\mathrm{CB}$ PVI procedure. The freedom of AF recurrence without AADs after a 13-month follow-up was obtained in $93 \%$ of patients. There was one more study including 18 patients with a paroxysmal lone AF referred to $\mathrm{CB}$ ablation (14). Throughout the mean follow-up of $14 \pm 9$ months, $78 \%$ of the patients were free of AF recurrence. It should be mentioned that in 2 patients a focal ablation catheter was needed to isolate the PVs.

Our recorded success rate without AADs in non-IAF patients at 1 and 3 years of follow-up was $64.6 \%$ and $39.9 \%$, respectively. When taking only paroxysmal AF into account, the freedom from atrial arrhythmias in this subgroup of patients at 1 and 3 years of follow-up was $72.6 \%$ and $47 \%$, respectively. In the recently published FREEZE Cohort study (6), 2329 patients with both paroxysmal and persistent AF were enrolled to the CB PVI arm. Following a single procedure, the incidence rates of symptomatic atrial arrhythmia recurrence on AADs were $30.7 \%$ after the median follow-up of 441 days. In STOP AF post-approval study (15), $64 \%$ of 344 paroxysmal AF patients treated with $\mathrm{CB}$ technique remained free from $\mathrm{AF}$ and symptomatic atrial arrhythmias after 36 months of follow-up. The efficacy results in our study are roughly consistent with the observations from the mentioned reports. However, the results cannot be directly comparable, since we considered both symptomatic and asymptomatic episodes of atrial arrhythmias, which were recorded, as a treatment failure. Moreover, in both of the above-mentioned studies, two balloon sizes or focal tip catheters were used to complete acute PVI in some patients. 
Several factors can be attributed to such good efficacy results for a single CB PVI procedure in IAF patients, especially those with paroxysmal AF. Most importantly, it is believed that IAF is more trigger-rather than substrate-dependent and as is generally known, these triggers are mainly located at the junction between LA and PVs (16). Another important fact is that these patients do not have such dilated LA, and thus a relatively large $28 \mathrm{~mm}$ balloon inflated in PV ostium can also capture the neighbouring areas of LA, which can also improve the ablation results (11).

According to the latest guidelines, there is a need to systematically report complications in clinical practice to improve the quality of AF ablation procedures (1). The most common adverse event of the CB PVI procedure is in general PNP. This was also observed in our study with an overall incidence of $3.9 \%$. However, most of PNPs was only transient and resolved spontaneously within the procedure. The incidence of major complications was $1.9 \%$, whereas only one case of pericardial effusion was life-threatening.

\section{Idiopathic atrial fibrillation}

In clinical practice, we encounter patients, who have AF without other clinically apparent associated risk factors such as: cardiovascular diseases, including structural heart diseases, cardiopulmonary diseases or endocrinopathies. The diagnosis of IAF is usually a diagnosis of exclusion. In this study, this diagnosis was made after a careful evaluation, including a consistent collection of patients medical history, echocardiography, ECG, physical examination, repeated blood pressure measurements, laboratory tests and CT of the heart. However, we are aware that some of the expected diseases may have been clinically latent or may not have been detected by routine screening methods. E.g. none of the IAF patients had confirmed coronary artery disease or had appropriate symptoms during follow-up, but the presence of silent myocardial ischemia cannot be completely excluded. Similarly, no IAF patient had a documented obstructive sleep apnoea, but, on the other hand, none of them had undergone a sleep laboratory testing.

In general, the number of IAF patients is not high but cannot be considered negligible. In the Euro Heart Survey, the prevalence was around $3 \%$ (17). Until recently, AF was referred to as "lone" in these patients. Currently, we are looking at the lone AF differently than in previous decades. It is believed that behind the onset of AF in these patients there are factors that are not detectable by conventional screening methods. Patients with lone AF have been reported to have mutations in a range of cardiac ion channels, structural proteins and signalling molecules (8).

According to the current guidelines, the historic term „lone AF" is misleading and should be avoided in nomenclature of AF (1). However, this group of patients still exists, so there is a need for a broader discussion of what term would be the best for these patients. In this study we use the term ,idiopathic" to address a specific group of AF patients, where we have not been able to identify the cause of the arrhythmia, although we believe that the cause exists.

Considering the current guidelines, genetic testing of AF patients is generally not recommended, as there is no clear link between any mutations found and therapeutic needs or specific outcome (1). Here, however, there are a few potential problems to be addressed. As have been published previously, many inherited channelopathies as well as monogenic defects are associated with AF even in younger populations (18-21). On the other hand, it is generally recommended to start treatment with AADs because randomized trials documented only modestly improved rhythm outcome with catheter ablation compared to drug therapy in the first line treatment of paroxysmal or asymptomatic AF (1, 22-23). When deciding to treat IAF patients regarding AADs vs. ablation, several important facts need to be considered. The first is the potential proarrhythmic effect of AADs, including lethal ventricular arrhythmias, which may occur in patients with a subclinical channelopathy predisposing to idiopathic AF treated with class I or III AADs $(11,24)$. The duration of AAD treatment in younger patients with further well-known complications, especially in the case of amiodaron, is also negligible. Considering these facts as well as promising efficiency of PVI there is a question arising, whether PVI by any technique may be appropriate as the first-line therapy in all IAF patients, even in the condition of paroxysmal or asymptomatic AF. Early intervention could prevent or at least slow down the progression of arrhythmia, which may also help to minimize or at least delay the need for AAD treatment.

\section{Limitations}

This study has some potential limitations. As was reported previously, the intermittent ECG monitoring is inferior to the continuous monitoring with implanted devices (25). This could lead to missing some asymptomatic or non-sustained episodes of atrial arrhythmias during follow-up.

In relation to IAF patients, we are fully aware that some of the monitored diseases predisposing to arrhythmia may have been clinically silent or unrecognized.

The definition of IAF we provide may differ somewhat from previous studies. This is a consequence of missing or not clearly formulated criteria on IAF/lone AF patients. We set the criteria for IAF in such a way that the diagnosis of IAF can be made on an outpatient basis relying on commonly available examination methods, blood tests and anamnestic data.

\section{Conclusions}

Based on the promising long-term efficacy results and low risk of severe complications, we assume that CBA PVI could become the first-line treatment that can minimize or at least delay the need for AADs in younger patients with idiopathic paroxysmal AF. Similar studies would be necessary to further expand the knowledge about the catheter treatment in this selected group of patients.

\section{References}

1. Kirchhof P, Benussi S, Kotecha D et al. 2016 ESC guidelines for the management of atrial fibrillation developed in collaboration with EACTS. Eur Heart J 2016; 37 (38): 2893-2962. 
89-94

2. Calkins H, Hindricks G, Cappato R et al. 2017 HRS/EHRA/ECAS/ APHRS/SOLAECE expert consensus statement on catheter and surgical ablation of atrial fibrillation: Executive summary. Europace 2018; 20 (1): 157-208

3. Irfan G, de Asmundis C, Mugnai G et al. One-year follow-up after second-generation cryoballoon ablation for atrial fibrillation in a large cohort of patients: A single-centre experience. Europace 2016; 18 (7): 987-993.

4. Kuck KH, Furnkranz A, Chun KR et al. Cryoballoon or radiofrequency ablation for symptomatic paroxysmal atrial fibrillation: reintervention, rehospitalization, and quality-of-life outcomes in the FIRE AND ICE trial. Eur Heart J 2016; 37 (38): 2858-2865.

5. Neumann T, Vogt J, Schumacher B et al. Circumferential pulmonary vein isolation with the cryoballoon technique: results from a prospective 3-center study. J Am Coll Cardiol 2008; 52 (4): 273-278.

6. Hoffmann E, Straube F, Wegscheider K et al. Outcomes of cryoballoon or radiofrequency ablation in symptomatic paroxysmal or persistent atrial fibrillation. Europace 2019; 21 (9): 1313-1324.

7. Evans W, Swann P. Lone auricular fibrillation. Br Heart J 1954; 16 (2): 189-194.

8. Wyse DG, Van Gelder IC, Ellinor PT et al. Lone atrial fibrillation: does it exist? J Am Coll Cardiol 2014; 63 (17): 1715-1723.

9. Chun KR, Schmidt B, Metzner A et al. The 'single big cryoballoon' technique for acute pulmonary vein isolation in patients with paroxysmal atrial fibrillation: a prospective observational single centre study. Eur Heart J 2009; 30 (6): 699-709.

10. Franceschi F, Dubuc M, Guerra PG et al. Diaphragmatic electromyography during cryoballoon ablation: a novel concept in the prevention of phrenic nerve palsy. Heart Rhythm 2011; 8 (6): 885-891.

11. Wójcik M, Berkowitsch A, Zaltsberg S et al. Cryoballoon ablation in young patients with lone paroxysmal atrial fibrillation. Rev Esp Cardiol (Engl Ed) 2014; 67 (7): 558-563.

12. Buiatti A, Kaess B, Reents T et al. Catheter Ablation for "Lone" Atrial Fibrillation: Efficacy and Predictors of Recurrence. J Cardiovasc Electrophysiol 2016; 27 (5): 536-541.

13. DE Regibus V, Mugnai G, Moran D et al. Second-Generation Cryoballoon Ablation in the Setting of Lone Paroxysmal Atrial Fibrillation: Single Procedural Outcome at 12 Months. J Cardiovasc Electrophysiol 2016; 27 (6): 677-682.
14. Namdar M, Chierchia GB, Westra S et al. Isolating the pulmonary veins as first-line therapy in patients with lone paroxysmal atrial fibrillation using the cryoballoon. Europace 2012; 14 (2): 197-203.

15. Knight BP, Novak PG, Sangrigoli R et al. Long-Term Outcomes After Ablation for Paroxysmal Atrial Fibrillation Using the Second-Generation Cryoballoon: Final Results From STOP AF Post-Approval Study. JACC Clin Electrophysiol 2019; 5 (3): 306-314.

16. Weerasooriya R, Khairy $\mathbf{P}$, Litalien $\mathbf{J}$ et al. Catheter ablation for atrial fibrillation: are results maintained at 5 years of follow-up? J Am Coll Cardiol 2011; 57 (2): 160-166.

17. Weijs B, Pisters R, Nieuwlaat $\mathbf{R}$ et al. Idiopathic atrial fibrillation revisited in a large longitudinal clinical cohort. Europace 2012; 14 (2): 184-190.

18. Johnson JN, Tester DJ, Perry J, Salisbury BA, Reed CR, Ackerman MJ. Prevalence of early-onset atrial fibrillation in congenital long QT syndrome. Heart Rhythm 2008; 5 (5): 704-709.

19. Zellerhoff S, Pistulli R, Mönnig G et al. Atrial Arrhythmias in longQT syndrome under daily life conditions: a nested case control study. J Cardiovasc Electrophysiol 2009; 20 (4): 401-407.

20. Darbar D, Kannankeril PJ, Donahue BS et al. Cardiac sodium channel (SCN5A) variants associated with atrial fibrillation. Circulation 2008; 117 (15): 1927-1935.

21. Ritchie MD, Rowan S, Kucera G et al. Chromosome 4q25 variants are genetic modifiers of rare ion channel mutations associated with familial atrial fibrillation. J Am Coll Cardiol 2012; 60 (13): 1173-1181.

22. Morillo CA, Verma A, Connolly SJ et al. Radiofrequency ablation vs antiarrhythmic drugs as first-line treatment of paroxysmal atrial fibrillation (RAAFT-2): a randomized trial. JAMA 2014; 311 (7): 692-700.

23. Hakalahti A, Biancari F, Nielsen JC, Raatikainen MJ. Radiofrequency ablation vs. antiarrhythmic drug therapy as first line treatment of symptomatic atrial fibrillation: systematic review and meta-analysis. Europace 2015 ; 17 (3): $370-378$.

24. Camm AJ. Safety considerations in the pharmacological management of atrial fibrillation. Int J Cardiol 2008; 127 (3): 299-306.

25. Verma A, Champagne J, Sapp J et al. Discerning the incidence of symptomatic and asymptomatic episodes of atrial fibrillation before and after catheter ablation (DISCERN AF): a prospective, multicenter study. JAMA Intern Med 2013; 173 (2): 149-156. 